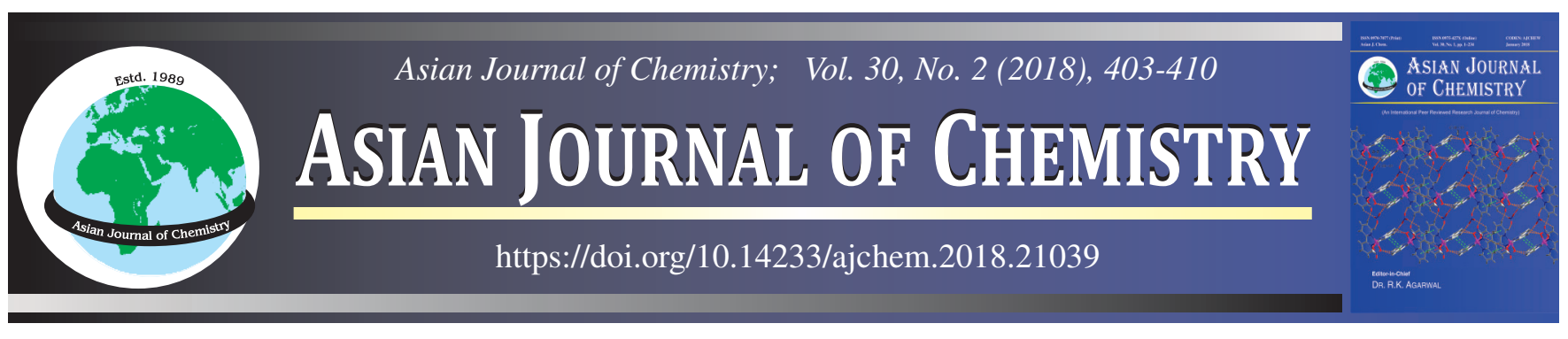

\title{
One Pot Multicomponent Synthesis of Anti-inflammatory Active Tetrahydrofuro[3,2-c]pyridinone-2-carboxylate Derivatives
}

\author{
Venkata Swamy Tangeti, ${ }^{1,2,}$, Boy Harika Reddy ${ }^{1}$ and K. Sharon Evangeline ${ }^{2}$
}

${ }^{1}$ Department of Chemistry, Raghu Engineering College, Visakhapatnam-531 162, India

${ }^{2}$ Vector Control Research Centre (Indian Council for Medical Research), Puducherry-605 006, India

*Corresponding author: E-mail: swamychempcu8@gmail.com

Received: 13 September 2017;

Accepted: 30 November 2017;

Published online: 31 December 2017;

AJC-18714

\begin{abstract}
A novel, one-pot method has been developed for the synthesis of tetrahydrofuro[3,2-c]pyridine-2-carboxylate derivatives via cascade four-component condensation of 6-methyl, 4-hydroxy pyranone, aryl amines, aromatic aldehydes and pyridinium ylide, in presence of triethylamine as a catalyst under ethanol reflux conditions. It affords the corresponding product in high yield (78-92\%) with short reaction time (15-25 $\mathrm{min})$.The anti-inflammatory activity of these compounds was determined. Out of the 17 synthesized compounds, compounds $\mathbf{5 j}$, 5k, 5l, 5m and $\mathbf{5 n}$ were excellent inhibitors of TNF- $\alpha$ and IL-6, COX-2, $\beta$-glucuronidase.
\end{abstract}

Keywords: 6-Methyl, 4-hydroxy pyranone, Pyridinium ylied, Multicomponent, Furopyridinone.

\section{INTRODUCTION}

The search for biologically active substances led to the investigation of condensed oxygen and nitrogen containing heterocycles. Furopyridines are very similar to such skeletons as quinoline and isoquinoline which are present in many compounds exhibiting biological activity [1]. Furo[3,2-c]pyridinone alkaloids are widespread among the Rutaceae family of plants and display important biological activities. In general furopyridone derivatives like citridone A, acridone alkaloid, dictamine, $\gamma$-fagarine, kimmianine, rutacridone-epoxide, hydroxyl rutacridone-epoxide shows good antibacterial activity (Fig. 1) [2-8].

Although a few synthetic approaches for the construction of this kind of heterocycle have been reported. Most of them may suffer from tedious steps, low yields and poor region and stereoselectivity [9-17]. Recently we have developed two new methodologies for the synthesis of C3-dihydrofuran substituted coumarins, dihydrofuro pyrazole by employing one pot multicomponent synthesis with pyridinium ylide. As part of our continued interest in the synthesis of diverse heterocyclic compounds of biological significance [18-25], we contemplated to synthesize novel tetrahydrofuro[3,2-c]pyridinone-2-carboxylate (5) by the one-pot four-component reaction of 6-methyl, 4-hydroxy pyran, aryl amine, aromatic aldehyde and pyridinium ylide to study their biological activity. Our previously developed methodology has been taken as a reference for developing furopyridone derivatives due to its slight structural similarity.<smiles>CC1=C[C@]2(C)Oc3c(-c4ccccc4)c[nH]c(=O)c3[C@H]2[C@H]1C</smiles>

Citrodone A<smiles>[R]c1ccc2c(OC)c3ccoc3nc2c1[R]</smiles>

Dictamine Gama-Fagarin
Kimmianine Rutacridone-Epoxide $\mathrm{R} 1=\mathrm{H}$

Fig. 1. Biologically active fuopyidene derivatives

Our present work has provided not only an efficient route to the structurally interesting and biologically significant furo[3,2$c$ ]pyridinone skeleton from readily available starting materials in a single step involves a tandem Michael addition fallowed by cyclization.

\section{EXPERIMENTAL}

Melting points were recorded using open-ended capillary tubes on VEEGO VMP-DS instrument. The progression of all 
the reactions was monitored by TLC using a mixture of hexanes (60-80 ${ }^{\circ} \mathrm{C}$ boiling mixture) and ethyl acetate. Column chromatography was performed on silica gel (100-200 mesh, SRL Chemicals) using increasing percentage of ethyl acetate in hexanes. ${ }^{1} \mathrm{H}$ NMR spectra (400 MHz) and ${ }^{13} \mathrm{C} \mathrm{NMR}(100 \mathrm{MHz})$ and DEPT-135 spectra were recorded for $\mathrm{CDCl}_{3}+\mathrm{CCl}_{4}(2: 1)$ solutions on a Bruker-400 spectrometer with tetramethylsilane (TMS) as internal standard; $J$ values are given in Hz. IR spectra were recorded as $\mathrm{KBr}$ solid solution on a Nicolet-6700 spectrometer. High resolution mass spectra were recorded on a Waters Micromass Q-TOF micro mass spectrometer using electron spray ionization mode. Organic solvents were distilled and dried before use. Melting points were measured in open capillary tubes and are uncorrected. Elemental analyses were performed on a Perkin Elmer 2400 Series II Elemental CHNS analyzer.

General procedure for synthesis of $(2 R, 3 R)$-ethyl 5-benzyl6-methyl-4-oxo-3-phenyl-2,3,4,5-tetrahydrofuro[3,2-c]pyridine-2-carboxylate (5a):A mixture of 4-hydroxy-6-methyl$2 H$-pyran-2-one (100 mg, $0.786 \mathrm{mmol})$, benzyl amine (84 mg, $0.786 \mathrm{mmol})$ in $\mathrm{EtOH}(5 \mathrm{~mL})$, was stirred at room temperature for $5 \mathrm{~min}$. Then benzaldehyde ( $83 \mathrm{mg}, 0.147 \mathrm{mmol}$ ), pyridinium ylide (193 mg, $0.786 \mathrm{mmol}$ ), triethylamine ( $8 \mathrm{mg}, 0.786 \mathrm{mmol}$ ) added sequentially to the mixture and it was refluxed at $80{ }^{\circ} \mathrm{C}$ for $20 \mathrm{~min}$ till the completion of the reaction (monitored by TLC). After completion of reaction ethanol was distilled out and in the product crushed ice $(25 \mathrm{~g}$ ) was added. To this $0.5 \mathrm{M}$ $\mathrm{HCl}(1 \mathrm{~mL})$ was added and the resulting aqueous with suspended solids was extracted with dichloromethane $(2 \times 10 \mathrm{~mL})$ and concentrated. Crude product was purified through column chromatography by eluting with hexanes and EtOAc mixtures (TLC, $45 \%$ EtOAc in hexanes; $\mathrm{R}_{\mathrm{f}}=0.34$ ). After recrystallization from $\mathrm{EtOH}$, product obtained as light yellow colour crystalline solid; Yield $86 \%$ (264 mg), m.p.: $154.3^{\circ} \mathrm{C}$, IR $\left(\mathrm{KBr}, \mathrm{v}_{\max }, \mathrm{cm}^{-1}\right)$ : 3015, 3002, 2995, 1789, 1680, 1622, 1502, 1208, 1180, 1073, 963, 870, 751; ${ }^{1} \mathrm{H}$ NMR (400 MHz, $\left.\mathrm{CDCl}_{3}\right) \delta 7.40(\mathrm{t}, J=7.8$ $\mathrm{Hz}, 2 \mathrm{H}), 7.33(\mathrm{t}, J=7.8 \mathrm{~Hz}, 2 \mathrm{H}), 7.29-7.23(\mathrm{~m}, 6 \mathrm{H}), 5.55(\mathrm{~s}$, $1 \mathrm{H}), 5.41(\mathrm{~d}, J=4.6 \mathrm{~Hz}, 1 \mathrm{H}), 4.98(\mathrm{~s}, 2 \mathrm{H}), 4.44(\mathrm{~d}, J=4.6 \mathrm{~Hz}$, $1 \mathrm{H}), 4.21$ (q, $J=6.6 \mathrm{~Hz}, 2 \mathrm{H}), 2.26(\mathrm{~s}, 3 \mathrm{H}), 1.29$ (t, $J=7.4 \mathrm{~Hz}$, $3 \mathrm{H}) \mathrm{ppm} ;{ }^{13} \mathrm{C}$ NMR $\left(100 \mathrm{MHz}, \mathrm{CDCl}_{3}\right) \delta 170.8,167.2,157.6$, 150.1, 140.6, 136.5, 128.6, 128.4, 127.9, 126.8, 126.5, 100.7, 100.4, 79.3, 61.6, 48.8, 40.8, 20.3, 14.1 ppm; HRMS (ESI, $m / z): 412.1519$ calcd. for $\mathrm{C}_{24} \mathrm{H}_{23} \mathrm{NO}_{4}(\mathrm{M}+\mathrm{Na})$ found: 412.1515 . Analysis calcd. for $\mathrm{C}_{24} \mathrm{H}_{23} \mathrm{NO}_{4}$ : C, 74.02; H, 5.95; N, 3.60; Found C, 74.01; H, 5.93; N, 3.58.

(2R,3R)-Ethyl 5-benzyl-3-(4-fluorophenyl)-6-methyl-4oxo-2,3,4,5-tetrahydrofuro[3,2-c]pyridine-2-carboxylate (5b): Yellow colour solid, Yield $89 \%$ (286 mg), m.p.: $157.5^{\circ} \mathrm{C}$, IR $\left(\mathrm{KBr}, v_{\max }, \mathrm{cm}^{-1}\right): 3011,3000,2981,1781,1676,1618,1500$, $1233,1191,1081,968,877,753 ;{ }^{1} \mathrm{H}$ NMR $\left(400 \mathrm{MHz}, \mathrm{CDCl}_{3}\right)$ $\delta 7.33-7.19(\mathrm{~m}, 9 \mathrm{H}), 5.55(\mathrm{~s}, 1 \mathrm{H}), 5.41(\mathrm{~d}, J=4.6 \mathrm{~Hz}, 1 \mathrm{H})$, $4.98(\mathrm{~s}, 2 \mathrm{H}), 4.44(\mathrm{~d}, J=4.6 \mathrm{~Hz}, 1 \mathrm{H}), 4.21(\mathrm{q}, J=6.6 \mathrm{~Hz}$, 2H), $2.26(\mathrm{~s}, 3 \mathrm{H}), 1.29$ (t, $J=7.4 \mathrm{~Hz}, 3 \mathrm{H}) \mathrm{ppm} ;{ }^{13} \mathrm{C}$ NMR $(100$ $\left.\mathrm{MHz}, \mathrm{CDCl}_{3}\right)$ 170.8, 167.3, 160.1, 157.6, 150.2, 136.5, 136.2, $129.3,128.5,126.9,126.7,115.4,100.7,100.4$, 79.4, 61.6, 48.7, 40.8, 20.3, 14.1 ppm; HRMS (ESI, $m / z)$ : 430.1425 calcd. for $\mathrm{C}_{24} \mathrm{H}_{22} \mathrm{FNO}_{4}(\mathrm{M}+\mathrm{Na})$ found: 430.1422. Analysis calcd. for $\mathrm{C}_{24} \mathrm{H}_{22} \mathrm{FNO}_{4}$ : C, 70.75; H, 5.44; N, 3.44; Found C, 70.73; H, $5.42 ; \mathrm{N}, 3.41$.
(2R,3R)-Ethyl 5-benzyl-3-(4-chlorophenyl)-6-methyl4-oxo-2,3,4,5-tetrahydrofuro[3,2-c]pyridine-2-carboxylate (5c) Yellow colour solid, Yield $92 \%$ (308 mg), m.p.: $157.9{ }^{\circ} \mathrm{C}$, IR $\left(\mathrm{KBr}, \mathrm{v}_{\max }, \mathrm{cm}^{-1}\right): 3015,3002,2995,1789,1680,1622,1502$, 1208, 1180, 1073, 963, 870, 751; ${ }^{1} \mathrm{H} \mathrm{NMR} \mathrm{(400} \mathrm{MHz,} \mathrm{CDCl}_{3}$ ) $\delta{ }^{1} \mathrm{H}$ NMR $\left(400 \mathrm{MHz}, \mathrm{CDCl}_{3}\right) \delta 7.44(\mathrm{~d}, J=7.8 \mathrm{~Hz}, 2 \mathrm{H}), 7.40$ $(\mathrm{d}, J=8.2 \mathrm{~Hz}, 2 \mathrm{H}), 7.33(\mathrm{t}, J=7.8 \mathrm{~Hz}, 3 \mathrm{H}), 7.28(\mathrm{t}, J=8.2$ $\mathrm{Hz}, 1 \mathrm{H}), 7.23$ (d, $J=8.2 \mathrm{~Hz}, 2 \mathrm{H}), 5.55(\mathrm{~s}, 1 \mathrm{H}), 5.41(\mathrm{~d}, J=4.6$ $\mathrm{Hz}, 1 \mathrm{H}), 4.97(\mathrm{~s}, 2 \mathrm{H}), 4.43$ (d, $J=4.6 \mathrm{~Hz}, 1 \mathrm{H}), 4.22$ (q, $J=6.6$ $\mathrm{Hz}, 2 \mathrm{H}), 2.25$ (s, 3H), 1.29 (t, $J=7.4 \mathrm{~Hz}, 3 \mathrm{H}) \mathrm{ppm} ;{ }^{13} \mathrm{C} \mathrm{NMR}$ $\left(100 \mathrm{MHz}, \mathrm{CDCl}_{3}\right) \delta 170.7,167.2,157.6,150.2,140.6,138.7$, 136.7, 131.6, 128.9, 128.5, 126.9, 126.7, 100.7, 100.4, 79.3, 61.6, 48.6, 40.8, 20.4, 14.1 ppm; HRMS (ESI, $m / z)$ : 423.1237 calcd. for $\mathrm{C}_{24} \mathrm{H}_{22} \mathrm{NO}_{4} \mathrm{Cl}(\mathrm{M}+\mathrm{Na})$ found: 423.1235. Analysis calcd. for $\mathrm{C}_{24} \mathrm{H}_{22} \mathrm{NO}_{4} \mathrm{Cl}$ : C, 68.00; H, 5.23; N, 3.30; Found C, 67.98; H, 5.21; N, 3.27.

(2R,3R)-Ethyl 5-benzyl-3-(4-bromophenyl)-6-methyl4-oxo-2,3,4,5-tetrahydrofuro[3,2-c]pyridine-2-carboxylate (5d): Yellow colour solid, Yield $91 \%(337 \mathrm{mg})$, m.p.: $158.9^{\circ} \mathrm{C}$, IR $\left(\mathrm{KBr}, \nu_{\max }, \mathrm{cm}^{-1}\right): 3021,3012,3003,1784,1691,1622,1610$, 1502, 1203, 1198, 1081, 955, 862, 722; ${ }^{1} \mathrm{H}$ NMR (400 MHz, $\left.\mathrm{CDCl}_{3}\right) \delta 7.94(\mathrm{~d}, J=8.2 \mathrm{~Hz}, 2 \mathrm{H}), 7.33(\mathrm{t}, J=7.6 \mathrm{~Hz}, 2 \mathrm{H})$, $7.29(\mathrm{t}, J=7.6 \mathrm{~Hz}, 2 \mathrm{H}), 7.23(\mathrm{~d}, J=8.2 \mathrm{~Hz}, 2 \mathrm{H}), 7.18(\mathrm{~d}, J=$ $7.6 \mathrm{~Hz}, 2 \mathrm{H}), 5.55(\mathrm{~s}, 1 \mathrm{H}), 5.41(\mathrm{~d}, J=4.6 \mathrm{~Hz}, 1 \mathrm{H}), 4.97$ (s, $2 \mathrm{H}), 4.44(\mathrm{~d}, J=4.6 \mathrm{~Hz}, 1 \mathrm{H}), 4.20(\mathrm{q}, J=6.6 \mathrm{~Hz}, 2 \mathrm{H}), 2.27(\mathrm{~s}$, $3 \mathrm{H}), 1.29$ (t, $J=7.4 \mathrm{~Hz}, 3 \mathrm{H}) \mathrm{ppm} ;{ }^{13} \mathrm{C} \mathrm{NMR}\left(100 \mathrm{MHz}, \mathrm{CDCl}_{3}\right)$ $\delta 170.8,167.2,157.6,150.1,139.8,136.5,131.5,129.9,128.5$, 126.9, 126.7, 120.5,100.7, 100.4, 79.3, 61.6, 48.7, 40.8, 20.4, 14.1 ppm; HRMS (ESI, $m / z$ ) : 490.0630 calcd. for $\mathrm{C}_{24} \mathrm{H}_{22} \mathrm{NO}_{4} \mathrm{Br}$ $\left(\mathrm{M}+\mathrm{Na}\right.$ ) found: 490.0626. Analysis calcd. for $\mathrm{C}_{24} \mathrm{H}_{22} \mathrm{NO}_{4} \mathrm{Br}$ : C, 61.55; H, 4.73; N, 2.99; Found C, 61.53; H, 4.71; N, 2.98.

$(2 R, 3 R)$-Ethyl 5-benzyl-6-methyl-4-oxo-3-p-tolyl2,3,4,5-tetrahydrofuro[3,2-c]pyridine-2-carboxylate (5e): Yellow colour solid, Yield $90 \%$ (287 mg), m.p.: $158.3^{\circ} \mathrm{C}$, IR $\left(\mathrm{KBr}, \mathrm{v}_{\max }, \mathrm{cm}^{-1}\right)$ : 3021, 3008, 3002, 2998, 1791, 1682, 1620, 1508, 1218, 1181, 1069, 952, 853, 775; ${ }^{1} \mathrm{H}$ NMR (400 MHz, $\left.\mathrm{CDCl}_{3}\right) \delta 7.33(\mathrm{t}, J=7.4 \mathrm{~Hz}, 2 \mathrm{H}), 7.28(\mathrm{~d}, J=7.4,2 \mathrm{H}), 7.23$ (t, $J=8.2 \mathrm{~Hz}, 1 \mathrm{H}), 7.17(\mathrm{~d}, J=8.2 \mathrm{~Hz}, 2 \mathrm{H}), 6.94(\mathrm{~d}, J=7.4$ $\mathrm{Hz}, 2 \mathrm{H}), 5.55$ (s, 1H), 5.41 (d, J = 4.6 Hz, 1H), 4.98 (s, 2H), $4.44(\mathrm{~d}, J=4.6 \mathrm{~Hz}, 1 \mathrm{H}), 4.22(\mathrm{q}, J=6.6 \mathrm{~Hz}, 2 \mathrm{H}), 2.34(\mathrm{~s}, 3 \mathrm{H})$, $2.16(\mathrm{~s}, 3 \mathrm{H}), 1.29$ (t, $J=7.4 \mathrm{~Hz}, 3 \mathrm{H}) \mathrm{ppm} ;{ }^{13} \mathrm{C}$ NMR $(100$ $\left.\mathrm{MHz}, \mathrm{CDCl}_{3}\right) \delta 170.8,167.2,157.6,150.1,137.6,136.5,135.6$, 128.9, 128.5, 127.6, 126.7, 100.7, 100.4, 79.3, 61.6, 48.5, 40.6, 21.3, 20.3, $14.1 \mathrm{ppm}$; HRMS (ESI, $\mathrm{m} / z$ ) : 426.1681 calcd. for $\mathrm{C}_{25} \mathrm{H}_{25} \mathrm{NO}_{4}(\mathrm{M}+\mathrm{Na})$ found: 426.1679. Analysis calcd. for $\mathrm{C}_{25} \mathrm{H}_{25} \mathrm{NO}_{4}$ : C, 74.42; H, 6.25; N, 3.47; Found C, 74.40; H, $6.22 ; \mathrm{N}, 3.44$.

(2R,3R)-Ethyl 5-benzyl-3-(4-methoxyphenyl)-6-methyl4-oxo-2,3,4,5-tetrahydrofuro[3,2-c]pyridine-2-carboxylate (5f): Yellow colour solid, Yield $90 \%$ (332 mg), m.p.: $159.1^{\circ} \mathrm{C}$, IR $\left(\mathrm{KBr}, \mathrm{v}_{\max }, \mathrm{cm}^{-1}\right)$ : 3023, 3015, 2995, 1798, 1675, 1614, 1500, 1204, 1172, 1062, 957, 865, 773; ${ }^{1} \mathrm{H}$ NMR (400 MHz, $\left.\mathrm{CDCl}_{3}\right) \delta 7.34(\mathrm{~d}, J=7.8 \mathrm{~Hz}, 2 \mathrm{H}), 7.30(\mathrm{t}, J=7.8 \mathrm{~Hz}, 2 \mathrm{H})$, $7.28(\mathrm{t}, J=7.8 \mathrm{~Hz}, 1 \mathrm{H}), 7.23(\mathrm{~d}, J=6.8 \mathrm{~Hz}, 2 \mathrm{H}), 6.94(\mathrm{~d}, J=$ $6.8 \mathrm{~Hz}, 2 \mathrm{H}), 5.55(\mathrm{~s}, 1 \mathrm{H}), 5.41(\mathrm{~d}, J=4.6 \mathrm{~Hz}, 1 \mathrm{H}), 4.98$ (s, $2 \mathrm{H}), 4.44(\mathrm{~d}, J=4.6 \mathrm{~Hz}, 1 \mathrm{H}), 4.21(\mathrm{q}, J=6.6 \mathrm{~Hz}, 2 \mathrm{H}), 3.83$ (s, $3 \mathrm{H}), 2.18$ (s, 3H), 1.29 (t, $J=7.4 \mathrm{~Hz}, 3 \mathrm{H}) \mathrm{ppm} ;{ }^{13} \mathrm{C} \mathrm{NMR}(100$ $\left.\mathrm{MHz}, \mathrm{CDCl}_{3}\right) \delta 170.7,167.2,157.8,157.6,150.1,136.5,132.9$, 
$128.9,128.9,128.7,128.5,126.9,126.7,114.2,100.4,79.3$ 61.6, 55.9, 48.6, 40.8, 20.3, $14.1 \mathrm{ppm}$; HRMS (ESI, $\mathrm{m} / \mathrm{z})$ : 442.1630 calcd. for $\mathrm{C}_{25} \mathrm{H}_{25} \mathrm{NO}_{5}(\mathrm{M}+\mathrm{Na})$ found: 442.1628 . Analysis calcd. for $\mathrm{C}_{25} \mathrm{H}_{25} \mathrm{NO}_{5}$ : C, 71.58; H, 6.01; N, 3.34; Found C, C, 71.55; H, 6.00; N, 3.32.

(2R,3R)-Ethyl 5-benzyl-3-(4-hydroxyphenyl)-6-methyl4-oxo-2,3,4,5-tetrahydrofuro[3,2-c]pyridine-2-carboxylate (5g): Yellow colour solid, Yield $92 \%$ (295 mg), m.p.: $157.6^{\circ} \mathrm{C}$, IR $\left(\mathrm{KBr}, \mathrm{v}_{\max }, \mathrm{cm}^{-1}\right): 3125,3081,3009,3001,2981,1764$, $1691,1618,1521,1321,1202,1178,1054,961,875,762 ;{ }^{1} \mathrm{H}$ NMR (400 MHz, $\left.\mathrm{CDCl}_{3}\right) \delta 7.33(\mathrm{~d}, J=7.8 \mathrm{~Hz}, 2 \mathrm{H}), 7.28(\mathrm{t}, J$ $=7.8 \mathrm{~Hz}, 2 \mathrm{H}), 7.24(\mathrm{t}, J=7.8 \mathrm{~Hz}, 1 \mathrm{H}), 7.23(\mathrm{~d}, J=6.8 \mathrm{~Hz}$, $2 \mathrm{H}), 6.70(\mathrm{~d}, J=6.8 \mathrm{~Hz}, 2 \mathrm{H}), 5.55(\mathrm{~s}, 1 \mathrm{H}), 5.41(\mathrm{~d}, J=4.6 \mathrm{~Hz}$, $1 \mathrm{H}), 5.23$ (br s, 1H), 4.98 (s, 2H), 4.44 (d, $J=4.6 \mathrm{~Hz}, 1 \mathrm{H})$, $4.21(\mathrm{q}, J=6.6 \mathrm{~Hz}, 2 \mathrm{H}), 2.26(\mathrm{~s}, 3 \mathrm{H}), 1.29(\mathrm{t}, J=7.4 \mathrm{~Hz}, 3 \mathrm{H})$ ppm; ${ }^{13} \mathrm{C}$ NMR $\left(100 \mathrm{MHz}, \mathrm{CDCl}_{3}\right) \delta 170.8,167.2,157.6$, $155.7,150.1,136.5,133.2,129.1,128.5,126.9,126.7,115.8$, 100.7, 100.4, 79.3, 61.6, 48.6, 40.8, 20.3, 14.1 ppm; HRMS (ESI, $m / z)$ : 428.1474 calcd. for $\mathrm{C}_{24} \mathrm{H}_{23} \mathrm{NO}_{5}(\mathrm{M}+\mathrm{Na})$ found: 428.1471. Analysis calcd. for $\mathrm{C}_{24} \mathrm{H}_{23} \mathrm{NO}_{5}$ : C, 71.10; H, 5.72; N, 3.45; Found C, 71.07; H, 5.70; N, 3.42.

(2R,3R)-Ethyl 5-benzyl-6-methyl-3-(4-nitrophenyl)-4oxo-2,3,4,5-tetrahydrofuro[3,2-c]pyridine-2-carboxylate (5h): Yellow colour solid, Yield $81 \%$ (278 mg), m.p.: $161.4{ }^{\circ} \mathrm{C}$, IR $\left(\mathrm{KBr}, \nu_{\max }, \mathrm{cm}^{-1}\right): 3034,3016,3005,2981,1776,1689,1614$, 1502, 1384, 1208, 1180, 1079, 971, 888, 753; ${ }^{1} \mathrm{H}$ NMR (400 $\left.\mathrm{MHz}, \mathrm{CDCl}_{3}\right) \delta 8.21(\mathrm{~d}, J=8.0 \mathrm{~Hz}, 2 \mathrm{H}), 7.55(\mathrm{~d}, J=8.0 \mathrm{~Hz}$, $2 \mathrm{H}), 7.33(\mathrm{~m}, 2 \mathrm{H}), 7.28(\mathrm{t}, J=7.6 \mathrm{~Hz}, 1 \mathrm{H}), 7.23(\mathrm{~d}, J=7.6$ $\mathrm{Hz}, 2 \mathrm{H}), 5.55$ (s, 1H), 5.41 (d, $J=4.6 \mathrm{~Hz}, 1 \mathrm{H}), 4.98(\mathrm{~s}, 2 \mathrm{H})$, $4.44(\mathrm{~d}, J=4.6 \mathrm{~Hz}, 1 \mathrm{H}), 4.21(\mathrm{q}, J=6.6 \mathrm{~Hz}, 2 \mathrm{H}), 2.26(\mathrm{~s}, 3 \mathrm{H})$, $1.29(\mathrm{t}, J=7.4 \mathrm{~Hz}, 3 \mathrm{H}) \mathrm{ppm} ;{ }^{13} \mathrm{C} \mathrm{NMR}\left(100 \mathrm{MHz}, \mathrm{CDCl}_{3}\right) \delta$ $170.8,167.2$, 157.6, 150.5, 147.2, 145.2, 136.5, 128.6, 128.5, 126.9, 126.8, 123.8, 100.7, 100.4, 79.4, 61.5, 48.7, 40.8, 20.3, 14.1 ppm; HRMS (ESI, $m / z$ ) : 457.1370 calcd. for $\mathrm{C}_{24} \mathrm{H}_{22} \mathrm{~N}_{2} \mathrm{O}_{6}$ $\left(\mathrm{M}+\mathrm{Na}\right.$ ) found: 457.1368. Analysis calcd. for $\mathrm{C}_{24} \mathrm{H}_{22} \mathrm{~N}_{2} \mathrm{O}_{6}$ : C, 66.35; H, 5.10; N, 6.45; Found C, 66.32; H, 5.08; N, 6.43.

(2R,3S)-Ethyl 5-benzyl-3-(2,4-dichlorophenyl)-6-methyl4-oxo-2,3,4,5-tetrahydrofuro[3,2-c]pyridine-2-carboxylate (5i): Yellow colour solid, Yield $83 \%$ (301 mg), m.p.: $166.7^{\circ} \mathrm{C}$, IR $\left(\mathrm{KBr}, \mathrm{v}_{\max }, \mathrm{cm}^{-1}\right): 3031,3022,3011,2987,1774,1698$, $1643,1621,1513,1209,1181,1079,974,878,758 ;{ }^{1} \mathrm{H}$ NMR $\left(400 \mathrm{MHz}, \mathrm{CDCl}_{3}\right) \delta 7.75(\mathrm{~s}, 1 \mathrm{H}), 7.33(\mathrm{~d}, J=6.8 \mathrm{~Hz}, 2 \mathrm{H})$, $7.30(\mathrm{~d}, J=7.0 \mathrm{~Hz}, 1 \mathrm{H}), 7.28(\mathrm{~m}, 1 \mathrm{H}), 7.23(\mathrm{~d}, J=7.6 \mathrm{~Hz}$, $2 \mathrm{H}), 7.17(\mathrm{~d}, J=7.6 \mathrm{~Hz}, 1 \mathrm{H}), 5.55(\mathrm{~s}, 1 \mathrm{H}), 5.41(\mathrm{~d}, J=4.6 \mathrm{~Hz}$, 1H), 4.98 (s, 1H), 4.44 (d, $J=4.6 \mathrm{~Hz}, 1 \mathrm{H}), 4.21$ (q, $J=6.6 \mathrm{~Hz}$, $3 \mathrm{H}), 2.76$ (s, 3H), 1.29 (t, $J=7.4 \mathrm{~Hz}, 3 \mathrm{H}) \mathrm{ppm} ;{ }^{13} \mathrm{C}$ NMR $(100$ $\left.\mathrm{MHz}, \mathrm{CDCl}_{3}\right) \delta 170.8,167.2,157.6,150.1,136.3,134.4,132.9$, $130.5,130.3,128.5,126.8,126.7,100.7,100.4,78.8,61.6$, 48.7, 35.8, 20.3, 14.1 ppm; HRMS (ESI, $\mathrm{m} / \mathrm{z}$ ) : 480.0740 calcd. for $\mathrm{C}_{24} \mathrm{H}_{21} \mathrm{NO}_{4} \mathrm{Cl}_{2}(\mathrm{M}+\mathrm{Na})$ found: 480.0738 . Analysis calcd. for $\mathrm{C}_{24} \mathrm{H}_{21} \mathrm{NO}_{4} \mathrm{Cl}_{2}$ : C, 62.89; H, 4.62; N, 3.06; Found C, 62.86; $\mathrm{H}, 4.60 ; \mathrm{N}, 3.04$.

(2R,3R)-Ethyl 5-benzyl-6-methyl-4-oxo-3-(2,4,5trimethoxyphenyl)-2,3,4,5-tetrahydrofuro[3,2-c]pyridine2-carboxylate (5j): Yellow colour solid, Yield $84 \%$ (373 mg), m.p.: $172.1^{\circ} \mathrm{C}$, IR $\left(\mathrm{KBr}, \nu_{\max }, \mathrm{cm}^{-1}\right): 3098,3065,3010,2982$, 1764, 1692, 1644, 1615, 1534, 1423, 1221, 1175, 1066, 951, 853, 742; ${ }^{1} \mathrm{H}$ NMR (400 MHz, $\left.\mathrm{CDCl}_{3}\right) \delta 7.33-7.29(\mathrm{~m}, 5 \mathrm{H})$, $6.85(\mathrm{~s}, 1 \mathrm{H}), 6.42(\mathrm{~s}, 1 \mathrm{H}), 5.55(\mathrm{~s}, 1 \mathrm{H}), 5.41(\mathrm{~d}, J=4.6 \mathrm{~Hz}$, $1 \mathrm{H}), 4.98(\mathrm{~s}, 2 \mathrm{H}), 4.44(\mathrm{~d}, J=4.6 \mathrm{~Hz}, 1 \mathrm{H}), 4.21(\mathrm{t}, J=6.6 \mathrm{~Hz}$, 2H), 3.83 (s, 9H), 2.96 (s, 3H), 1.29 (t, $J=7.4 \mathrm{~Hz}, 3 \mathrm{H}) \mathrm{ppm}$; ${ }^{13} \mathrm{C}$ NMR $\left(100 \mathrm{MHz}, \mathrm{CDCl}_{3}\right) \delta 170.8,167.2,157.6,150.1$, $148.8,144.5,136.5,128.5,126.9,126.7,119.5,111.1,100.7$, 100.4, 100.1, 79.6, 61.6, 56.13, 56.12, 56.11, 48.7, 35.2, 20.3, 14.1 ppm; HRMS (ESI, $m / z$ ) : 502.1842 calcd. for $\mathrm{C}_{27} \mathrm{H}_{29} \mathrm{NO}_{7}$ $\left(\mathrm{M}+\mathrm{Na}\right.$ ) found: 502.1840. Analysis calcd. for $\mathrm{C}_{27} \mathrm{H}_{29} \mathrm{NO}_{7}$ : $\mathrm{C}$, 67.63; H, 6.10; N, 2.92; Found C, 67.63; H, 6.10; N, 2.92.

(2R,3R)-Ethyl 5-(4-methoxybenzyl)-6-methyl-4-oxo-3(2,4,5-trimethoxyphenyl)-2,3,4,5-tetrahydrofuro[3,2c]pyridine-2-carboxylate (5k): Yellow colour solid, Yield 85 $\%$ (342 mg), m.p.: $175.4^{\circ} \mathrm{C}$, IR (KBr, $\left.v_{\max }, \mathrm{cm}^{-1}\right)$ : 3025, 3014, 3004, 2985, 1763, 1674, 1618, 1501, 1211, 1158, 1154,1073, 963, 870, 842, 744; ${ }^{1} \mathrm{H}$ NMR (400 MHz, $\left.\mathrm{CDCl}_{3}\right) \delta 7.83(\mathrm{~s}$, $1 \mathrm{H}), 7.57$ (s, 1H), 7.25 (d, $J=6.8 \mathrm{~Hz}, 2 \mathrm{H}), 6.87$ (d, $J=6.8 \mathrm{~Hz}$, 2H), $5.55(\mathrm{~s}, 1 \mathrm{H}), 5.41(\mathrm{~d}, J=4.6 \mathrm{~Hz}, 1 \mathrm{H}), 4.98(\mathrm{~s}, 2 \mathrm{H}), 4.44$ $(\mathrm{d}, J=4.6 \mathrm{~Hz}, 1 \mathrm{H}), 4.21(\mathrm{q}, J=6.6 \mathrm{~Hz}, 2 \mathrm{H}), 3.84(\mathrm{~s}, 3 \mathrm{H}), 2.26$ (s, 3H), 1.29 (t, $J=7.4 \mathrm{~Hz}, 3 \mathrm{H}) \mathrm{ppm} ;{ }^{13} \mathrm{C} \mathrm{NMR}(100 \mathrm{MHz}$, $\left.\mathrm{CDCl}_{3}\right) \delta 170.8,167.5,158.7,157.6,150.1,138.4,130.5$, $128.8,128.5,128.3,126.8,124.4,124.3,123.0,122.3,114.1$, 100.7, 100.4, 79.6, 61.6, 55.8, 48.7, 38.3, 20.3, 14.1 ppm; HRMS (ESI, m/z) : 532.1942 calcd. for $\mathrm{C}_{28} \mathrm{H}_{31} \mathrm{NO}_{8}(\mathrm{M}+\mathrm{Na})$ found: 532.1941. Analysis calcd. for $\mathrm{C}_{28} \mathrm{H}_{31} \mathrm{NO}_{8}: \mathrm{C}, 66.00 ; \mathrm{H}$, $6.13 ;$ N, 2.75; Found C, 65.98; H, 6.10; N, 2.72.

(2R,3R)-Ethyl 3-[2,4-bis(trifluoromethyl)phenyl]-5-(4methoxybenzyl)-6-methyl-4-oxo-2,3,4,5-tetrahydrofuro[3,2-c]pyridine-2-carboxylate (5l): Yellow colour solid, Yield $79 \%$ (304 mg), m.p.: $175.5^{\circ} \mathrm{C}$, IR ( KBr, $\left.v_{\max }, \mathrm{cm}^{-1}\right)$ : 3112 , 3023, 3004, 2991, 1765, 1684, 1620, 1543, 1226, 1180, 1115, 1064, 961, 864, 744; ${ }^{1} \mathrm{H}$ NMR (400 MHz, $\left.\mathrm{CDCl}_{3}\right) \delta 7.57$ (d, $J=8.2 \mathrm{~Hz}, 2 \mathrm{H}), 7.22(\mathrm{~d}, J=8.2 \mathrm{~Hz}, 2 \mathrm{H}), 7.25(\mathrm{~d}, J=6.8 \mathrm{~Hz}$, $2 \mathrm{H}), 6.87(\mathrm{~d}, J=6.8 \mathrm{~Hz}, 2 \mathrm{H}), 5.55(\mathrm{~s}, 1 \mathrm{H}), 5.41(\mathrm{~d}, J=4.6 \mathrm{~Hz}$, $1 \mathrm{H}), 4.98(\mathrm{~s}, 2 \mathrm{H}), 4.44(\mathrm{~d}, J=4.6 \mathrm{~Hz}, 1 \mathrm{H}), 4.21$ (q, $J=6.6$ $\mathrm{Hz}, 2 \mathrm{H}), 3.83$ (s, 3H), 2.26 (s, 3H), 1.29 (t, $J=7.4 \mathrm{~Hz}, 3 \mathrm{H})$ ppm; ${ }^{13} \mathrm{C}$ NMR $\left(100 \mathrm{MHz}, \mathrm{CDCl}_{3}\right) \delta 170.5,167.5,158.8$, $157.6,150.1,143.9,130.5,128.8,128.2,128.0,125.0,124.1$, $114.1,100.7,100.4,79.3,61.6,55.8,48.7,40.8,20.3$, $14.1 \mathrm{ppm}$; HRMS (ESI, $\mathrm{m} / z)$ ) 510.1499 calcd. for $\mathrm{C}_{26} \mathrm{H}_{24} \mathrm{NO}_{5} \mathrm{~F}_{3}(\mathrm{M}+\mathrm{Na})$ found: 510.1494. Analysis calcd. for $\mathrm{C}_{26} \mathrm{H}_{24} \mathrm{NO}_{5} \mathrm{~F}_{3}$ : C, 64.06; H, 4.96; N, 2.87; Found C, 64.04; H, $4.95 ; \mathrm{N}, 2.83$.

(2R, 3R)-Ethyl 3-(2,4-bis(trifluoromethyl)phenyl)-5-(4methoxybenzyl)-6-methyl-4-oxo-2,3,4,5-tetrahydrofuro[3,2-c]pyridine-2-carboxylate $(\mathbf{5 m})$ : Yellow colour solid, Yield $78 \%$ (343 mg), m.p.: $176.5^{\circ} \mathrm{C}$, IR $\left(\mathrm{KBr}, v_{\max }, \mathrm{cm}^{-1}\right)$ : 3042, 3014, 2987, 1792, 1692, 1618, 1523, 1218, 1184, 1082, 942, 854, $763 ;{ }^{1} \mathrm{H}$ NMR (400 MHz, $\left.\mathrm{CDCl}_{3}\right) \delta 7.57$ (d, $J=8.0$ $\mathrm{Hz}, 2 \mathrm{H}), 7.22$ (d, $J=8.0 \mathrm{~Hz}, 2 \mathrm{H}), 7.25$ (d, $J=6.8 \mathrm{~Hz}, 2 \mathrm{H})$, $6.87(\mathrm{~d}, J=6.8 \mathrm{~Hz}, 2 \mathrm{H}), 5.55(\mathrm{~s}, 1 \mathrm{H}), 5.41(\mathrm{~d}, J=1 \mathrm{H}), 4.98$ (s, $2 \mathrm{H}), 4.44(\mathrm{~d}, J=4.6 \mathrm{~Hz}, 1 \mathrm{H}), 4.21(\mathrm{q}, J=6.6 \mathrm{~Hz}, 2 \mathrm{H}), 3.83$ (s, $3 \mathrm{H}), 2.26$ (s, 3H), 1.29 (t, $J=7.4 \mathrm{~Hz}, 3 \mathrm{H}) \mathrm{ppm} ;{ }^{13} \mathrm{C} \mathrm{NMR}(100$ $\left.\mathrm{MHz}, \mathrm{CDCl}_{3}\right) \delta 170.5,167.5,158.8,157.6,150.1,143.9,130.5$, $128.8,128.2,128.0,125.0,124.1,114.1,100.7,100.4,79.3$, 61.6, 55.8, 48.7, 40.8, 20.3 ppm; HRMS (ESI, $m / z)$ : 578.1373 calcd. for $\mathrm{C}_{27} \mathrm{H}_{23} \mathrm{NO}_{5} \mathrm{~F}_{6}(\mathrm{M}+\mathrm{Na})$ found: 578.1370. Analysis calcd. for $\mathrm{C}_{27} \mathrm{H}_{23} \mathrm{NO}_{5} \mathrm{~F}_{6}$ : C, 58.38; H, 4.17; N, 2.52; Found C, 58.35; H, 4.14; N, 2.51. 
(2R,3R)-Ethyl 3-[2,4-bis(trifluoromethyl)phenyl]-5(3,4-dimethoxybenzyl)-6-methyl-4-oxo-2,3,4,5-tetrahydrofuro[3,2-c]pyridine-2-carboxylate (5n): Yellow colour solid, Yield $83 \%$ (384 mg), m.p.: $177.3^{\circ} \mathrm{C}$, IR $\left(\mathrm{KBr}, v_{\max }, \mathrm{cm}^{-1}\right)$ : 3124, 3016, 3011, 2985, 1795, 1692, 1634, 1532, 1202, 1171, 1063, 958, 854, $745 ;{ }^{1} \mathrm{H}$ NMR (400 MHz, $\left.\mathrm{CDCl}_{3}\right) \delta 7.83(\mathrm{~s}$, $1 \mathrm{H}), 7.57(\mathrm{~d}, \mathrm{~J}=8.2 \mathrm{~Hz}, 1 \mathrm{H}), 7.46(\mathrm{~d}, J=8.2 \mathrm{~Hz}, 1 \mathrm{H}), 7.32$ $(\mathrm{d}, J=6.8 \mathrm{~Hz}, 1 \mathrm{H}) 7.15(\mathrm{~d}, J=8.2 \mathrm{~Hz}, 1 \mathrm{H}), 6.82(\mathrm{~d}, J=6.8$ $\mathrm{Hz}, 1 \mathrm{H}), 5.55(\mathrm{~s}, 1 \mathrm{H}), 5.41(\mathrm{~d}, J=1 \mathrm{H}), 4.98(\mathrm{~s}, 2 \mathrm{H}), 4.44(\mathrm{~d}$, $J=1 \mathrm{H}), 4.21(\mathrm{q}, J=2 \mathrm{H}), 3.83(\mathrm{~s}, 3 \mathrm{H}), 3.82(\mathrm{~s}, 3 \mathrm{H}), 2.76(\mathrm{~s}$, $3 \mathrm{H}), 1.29(\mathrm{t}, J=3 \mathrm{H}) \mathrm{ppm} ;{ }^{13} \mathrm{C} \mathrm{NMR}\left(100 \mathrm{MHz}, \mathrm{CDCl}_{3}\right) \delta$ $170.8,167.2,158.6,157.6,150.1,138.4,130.5,128.8,128.5$, $128.3,126.8,124.4,124.3,123.0,122.3,114.1,100.7,100.4$, 78.9, 61.7, 55.8, 48.7, 38.3, 20.3, 14.1 ppm; HRMS (ESI, $m / z)$ : 608.1478 calcd. for $\mathrm{C}_{28} \mathrm{H}_{25} \mathrm{NO}_{6} \mathrm{~F}_{6}(\mathrm{M}+\mathrm{Na})$ found: 608.1475 . Analysis calcd. for $\mathrm{C}_{28} \mathrm{H}_{25} \mathrm{NO}_{6} \mathrm{~F}_{6}$ : C, 57.44; H, 4.30; N, 2.39; Found C, 57.42; H, 4.28; N, 2.36.

(2R,3R)-Ethyl 5-benzyl-3-(furan-2-yl)-6-methyl-4-oxo2,3,4,5-tetrahydrofuro[3,2-c]pyridine-2-carboxylate (5o): Yellow colour solid, Yield $88 \%$ (264 mg), m.p.: $168.8^{\circ} \mathrm{C}$, IR $\left(\mathrm{KBr}, \mathrm{v}_{\max }, \mathrm{cm}^{-1}\right): 3114,3015,1774,1684,1638,1622,1604$, 1521, 1432, 1217, 1179, 1087, 968, 864, 774; ${ }^{1} \mathrm{H}$ NMR (400 $\left.\mathrm{MHz}, \mathrm{CDCl}_{3}\right) \delta 7.58(\mathrm{~d}, J=8.6 \mathrm{~Hz}, 1 \mathrm{H}), 7.33(\mathrm{~m}, 2 \mathrm{H}), 7.27-$ $7.23(\mathrm{~m}, 3 \mathrm{H}), 6.40(\mathrm{t}, J=8.4 \mathrm{~Hz}, 1 \mathrm{H}), 6.08(\mathrm{~d}, J=8.4 \mathrm{~Hz}$, $1 \mathrm{H}), 5.55(\mathrm{~s}, 1 \mathrm{H}), 5.41(\mathrm{~d}, J=1 \mathrm{H}), 4.98(\mathrm{~s}, 2 \mathrm{H}), 4.67(\mathrm{~d}, J=$ $1 \mathrm{H}), 4.21(\mathrm{q}, J=2 \mathrm{H}), 2.26(\mathrm{~s}, 3 \mathrm{H}), 1.29(\mathrm{t}, J=3 \mathrm{H}) \mathrm{ppm} ;{ }^{13} \mathrm{C}$ NMR $\left(100 \mathrm{MHz}, \mathrm{CDCl}_{3}\right) \delta 170.8,167.2,157.6,151.6,150.1$, $141.5,136.5,128.7,126.9,126.7,110.0,105.9,100.7,100.4$, 77.5, 61.6, 48.7, 40.5, 20.3, $14.1 \mathrm{ppm}$; HRMS (ESI, $\mathrm{m} / \mathrm{z}$ ) : 379.1420 calcd. for $\mathrm{C}_{30} \mathrm{H}_{22} \mathrm{~N}_{2} \mathrm{O}_{6} \mathrm{~F}_{6}(\mathrm{M}+\mathrm{Na})$ found: 379.1416 . Analysis calcd. for $\mathrm{C}_{30} \mathrm{H}_{22} \mathrm{~N}_{2} \mathrm{O}_{6} \mathrm{~F}_{6}$ : C, 69.64; H, 5.58; N, 3.69; Found C, 69.62; H, 5.55; N, 3.65.

(2R,3R)-Ethyl 5-benzyl-6-methyl-4-oxo-3-(thiophen-2yl)-2,3,4,5-tetrahydrofuro[3,2-c]pyridine-2-carboxylate (5p): Yellow colour solid, Yield $87 \%(272 \mathrm{mg})$, m.p.: $169.2^{\circ} \mathrm{C}$, IR $\left(\mathrm{KBr}, \mathrm{v}_{\max }, \mathrm{cm}^{-1}\right): 3076,3056,3012,1799,1696,1646$, 1612, 1546, 1317, 1208, 1178, 1089, 989, 888, $779 ;{ }^{1} \mathrm{H}$ NMR $\left(400 \mathrm{MHz}, \mathrm{CDCl}_{3}\right) \delta 7.42(\mathrm{~d}, J=8.6 \mathrm{~Hz}, 1 \mathrm{H}), 7.33(\mathrm{t}, J=7.6$ $\mathrm{Hz}, 2 \mathrm{H}), 7.29$ (t, $J=7.6 \mathrm{~Hz}, 1 \mathrm{H}), 7.21(\mathrm{~d}, J=8.4 \mathrm{~Hz}, 2 \mathrm{H})$, $6.93(\mathrm{t}, J=8.2 \mathrm{~Hz}, 1 \mathrm{H}), 6.83(\mathrm{~d}, J=8.4 \mathrm{~Hz}, 1 \mathrm{H}), 5.55(\mathrm{~s}, 1 \mathrm{H})$, $5.41(\mathrm{~d}, J=4.6 \mathrm{~Hz}, 1 \mathrm{H}), 4.98(\mathrm{~s}, 1 \mathrm{H}), 4.44(\mathrm{~d}, J=4.6 \mathrm{~Hz}, 1 \mathrm{H})$, $4.21(\mathrm{q}, J=6.6 \mathrm{~Hz}, 2 \mathrm{H}), 2.26(\mathrm{~s}, 3 \mathrm{H}), 1.29(\mathrm{t}, J=7.4 \mathrm{~Hz}, 3 \mathrm{H})$ ppm; ${ }^{13} \mathrm{C}$ NMR $\left(100 \mathrm{MHz}, \mathrm{CDCl}_{3}\right) \delta 170.8,167.2,157.6$, $150.1,139.4,136.5,128.5,127.1,127.0,126.7,126.6,125.5$, 100.7, 100.4, 80.0, 61.6, 48.7, 40.0, 20.3, 14.1 ppm; HRMS (ESI, $m / z)$ : 395.1191 calcd. for $\mathrm{C}_{22} \mathrm{H}_{21} \mathrm{NO}_{4} \mathrm{~S}(\mathrm{M}+\mathrm{Na})$ found: 395.1189. Analysis calcd. for $\mathrm{C}_{22} \mathrm{H}_{21} \mathrm{NO}_{4} \mathrm{~S}$ : C, 66.82; H, 5.35; N, 3.54; S, 8.11; Found C, 66.80; H, 5.33; N, 3.52; S, 8.10.

(2R,3R)-Ethyl 5-benzyl-6-methyl-4-oxo-3-(pyridin-3yl)-2,3,4,5-tetrahydrofuro[3,2-c]pyridine-2-carboxylate (5q): Yellow colour solid, Yield $91 \%(281 \mathrm{mg})$, m.p.: $168.5^{\circ} \mathrm{C}$, IR $\left(\mathrm{KBr}, \mathrm{v}_{\max }, \mathrm{cm}^{-1}\right): 3078,3012,3001,1765,1675,1646$, 1602, 1497, 1232, 1196, 1077, 954, 881, $767 ;{ }^{1} \mathrm{H}$ NMR (400 $\left.\mathrm{MHz}, \mathrm{CDCl}_{3}\right) \delta 8.41(\mathrm{~s}, 1 \mathrm{H}), 7.67(\mathrm{~d}, J=7.8 \mathrm{~Hz}, 1 \mathrm{H}), 7.33$ $7.28(\mathrm{~m}, 6 \mathrm{H}), 5.55(\mathrm{~s}, 1 \mathrm{H}), 5.41(\mathrm{~d}, J=4.6 \mathrm{~Hz}, 1 \mathrm{H}), 4.98(\mathrm{~s}$, $2 \mathrm{H}), 4.44(\mathrm{~d}, J=4.6 \mathrm{~Hz}, 1 \mathrm{H}), 4.21(\mathrm{q}, J=6.6 \mathrm{~Hz}, 2 \mathrm{H}), 2.26$ (s, $3 \mathrm{H}), 1.29(\mathrm{t}, J=7.4 \mathrm{~Hz}, 3 \mathrm{H}) \mathrm{ppm} ;{ }^{13} \mathrm{C} \mathrm{NMR}\left(100 \mathrm{MHz}, \mathrm{CDCl}_{3}\right)$ $\delta 170.8,167.4,157.8,150.7,148.4,146.9,139.1,136.5,133.5$,
128.6, 126.9, 126.7, 123.6, 100.7, 100.4, 79.3, 61.8, 48.9, 40.9, 20.5, 14.5 ppm; HRMS (ESI, $\mathrm{m} / \mathrm{z}$ ): 413.1477 calcd. for $\mathrm{C}_{23} \mathrm{H}_{22} \mathrm{~N}_{2} \mathrm{O}_{4}(\mathrm{M}+\mathrm{Na})$ found: 413.1473. Analysis calcd. for $\mathrm{C}_{23} \mathrm{H}_{22} \mathrm{~N}_{2} \mathrm{O}_{4}$ : C, 70.75; H, 5.68; N, 7.17; Found C, 70.73; H, $5.65 ; \mathrm{N}, 7.16$.

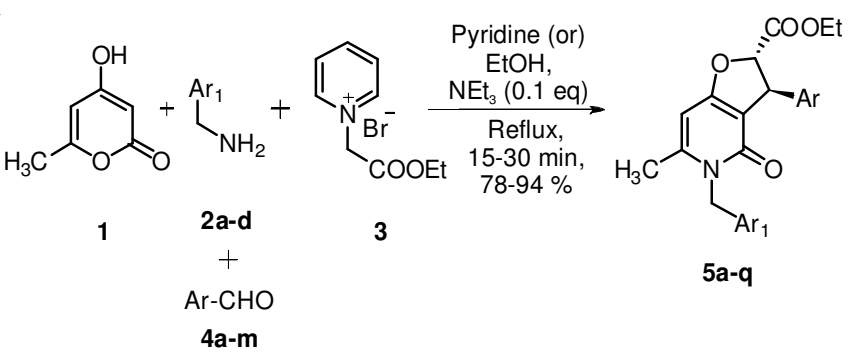

Scheme-I: Synthesis of tetrahydrofuro[3,2-c]pyridinone-2-carboxylate derivatives (5a-q)

\section{RESULTS AND DISCUSSION}

Initially, the condensation of with 6-methyl, 4-hydroxy pyran (1), aromatic aldehyde (2) pyridinium ylide (3) and aryl amine (4), was chosen as a model reaction and the influence of various solvents and catalysts was examined to find the optimal conditions for the reaction. In the first instance the reaction was investigated in the absence of catalyst (Table-1, entry 1) under solvent free microwave mediated conditions, but no product was formed. Then we decided to perform the reaction in suitable solvents under conventional heating methods. When the reaction was carried out in the presence of catalytic amount of $\mathrm{NEt}_{3}$ under ethanol solvent conditions, the reaction was occurred within $15 \mathrm{~min}$. Moreover, the nature of a base also has a pronounced impact on the reaction, $\mathrm{NEt}_{3}$ appears to be better than morpholine, L-proline, piperidene, $\mathrm{K}_{2} \mathrm{CO}_{3}$. On further modification of the reaction conditions, we were delighted to find that the usage of stoichiometric quantity of $\mathrm{NEt}_{3}$ was not necessary, only catalytic amount $(0.1$ eq.) was sufficient to effect this transformation efficiently. Next, we examined the effect of different solvents, which included $\mathrm{MeOH}$, water, $\mathrm{CH}_{2} \mathrm{Cl}_{2}$, THF, $\mathrm{CH}_{3} \mathrm{CN}$, DMF, toluene, $\mathrm{CHCl}_{3}$ under same reaction conditions (entries 12-21, Table-1). Among various solvents tested, EtOH was found to be the best solvent for the reaction, in terms of rapid transformation and quantified yields.

The structure of the compound was characterized by ${ }^{1} \mathrm{H}$ and ${ }^{13} \mathrm{C}$ NMR, MS and IR spectra and elemental analysis. In the ${ }^{1} \mathrm{H}$ NMR spectra, the two protons at 2,3-position of dihydrofuran ring display two doublets at 5.41 and $4.44 \mathrm{ppm}$ with the vicinal coupling constant $J=4.6$ and $4.6 \mathrm{~Hz}$, respectively. It has been documented that in cis-2,3-dihydrofuran the vicinal coupling constant of the two methine protons $J=7-10 \mathrm{~Hz}$, while in trans-2,3-dihydrofuran vicinal coupling constant $J=$ 4-7 Hz (Fig. 2). It is concluded that thermodynamically stable trans isomer of 2, 3-dihydrofuran derivatives were formed on pyridone moiety [26]. Stereochemical disposition of trans H's were confirmed by 2D NMR vis HSQC and HMBC. As anticipated all the 19 signals are appeared in ${ }^{13} \mathrm{C}$ NMR spectrum. HRMS spectra shows molecular ion $(\mathrm{M}+\mathrm{Na})$ peak at 412.1515. 
TABLE-1

OPTIMIZATION OF REACTION CONDITIONS

\begin{tabular}{|c|c|c|c|c|}
\hline Entry & Base $^{a}$ & Solvent & Time (min) & Yield $(\%)^{\mathrm{b}}$ \\
\hline 1 & No & No & $10 \mathrm{~min}$ & 0 \\
\hline 2 & Piperizene & No & $20 \mathrm{~min}$ & 28 \\
\hline 3 & Mapholine & No & $30 \mathrm{~min}$ & 37 \\
\hline 4 & DBU & No & $30 \mathrm{~min}$ & 16 \\
\hline 5 & $\mathrm{~K}_{2} \mathrm{CO}_{3}$ & No & $25 \mathrm{~min}$ & 39 \\
\hline 6 & Piperidene & No & $20 \mathrm{~min}$ & 42 \\
\hline 7 & Piperidene & water & $25 \mathrm{~min}$ & 57 \\
\hline 8 & Piperidene & Ethanol & $25 \min$ & 61 \\
\hline 8 & $\mathrm{NHEt}_{2}$ & No & $20 \mathrm{~min}$ & 57 \\
\hline 9 & $\mathrm{NEt}_{3}$ & No & $15 \mathrm{~min}$ & 75 \\
\hline 10 & $\mathrm{NEt}_{3}$ & $\mathrm{EtOH}$ & $30 \mathrm{~min}$ & 86 \\
\hline 11 & $\mathrm{NEt}_{3}$ & Water & $35 \mathrm{~min}$ & 65 \\
\hline 12 & $\mathrm{NEt}_{3}$ & DD Water & $40 \mathrm{~min}$ & 72 \\
\hline 12 & $\mathrm{NEt}_{3}$ & DMF & $70 \mathrm{~min}$ & 60 \\
\hline 13 & $\mathrm{NEt}_{3}$ & $\mathrm{MeCN}$ & $60 \mathrm{~min}$ & 56 \\
\hline 14 & $\mathrm{NEt}_{3}$ & THF & $3.5 \mathrm{~h}$ & Trace \\
\hline 15 & $\mathrm{NEt}_{3}$ & $\mathrm{CH}_{2} \mathrm{Cl}_{2}$ & $2.5 \mathrm{~h}$ & Trace \\
\hline 16 & $\mathrm{NEt}_{3}$ & Toluene & $2.0 \mathrm{~h}$ & Trace \\
\hline 17 & $\mathrm{NEt}_{3}$ & $\mathrm{CHCl}_{3}$ & $2.0 \mathrm{~h}$ & Trace \\
\hline
\end{tabular}

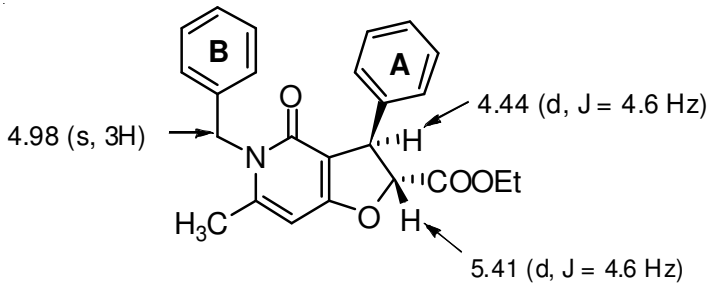

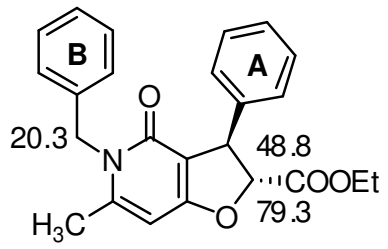

Fig. 2. Structure of tetrahydrofuro[3,2-c]pyridine-2-carboxylate

By using the above the optimized reaction conditions, we subjected a series of aromatic aldehyde derivatives, benzylamine derivatives for this protocol to explore the generality of substrate scope of the process and the results are summarized in Table- 2 . In almost all cases, the furo pyridone derivatives formation was quick and in good yields. Substitution of various ring substituted aromatic aldehyde derivatives, benzylamine derivatives with hydrazine and pyridinium ylide afforded pyrazole spiro chromanones 5a-q in 78-94 \% (Table-2, entries 1-17). Spectroscopic data of the products matched well with those of the parent compound 5a.

\begin{tabular}{|c|c|c|c|c|}
\hline \multicolumn{5}{|c|}{$\begin{array}{c}\text { TABLE-2 } \\
\text { SYNTHESIS OF TETRAHYDROFURO[3,2-c]- } \\
\text { PYRIDINONE-2-CARBOXYLATE }(\mathbf{5 a - q})\end{array}$} \\
\hline Entry & Compd. & $\mathrm{Ar}$ & $\mathrm{Ar}_{1}$ & $\begin{array}{l}\text { Yield } \\
(\%)^{c}\end{array}$ \\
\hline 1 & $5 a$ & $\mathrm{C}_{6} \mathrm{H}_{5}$ & $\mathrm{C}_{6} \mathrm{H}_{5}$ & 86 \\
\hline 2 & $5 \mathbf{b}$ & $\mathrm{C}_{6} \mathrm{H}_{4} \mathrm{Cl}$ & $\mathrm{C}_{6} \mathrm{H}_{5}$ & 89 \\
\hline 3 & $5 c$ & $\mathrm{C}_{6} \mathrm{H}_{4} \mathrm{Br}$ & $\mathrm{C}_{6} \mathrm{H}_{5}$ & 92 \\
\hline 4 & $5 d$ & $\mathrm{C}_{6} \mathrm{H}_{4} \mathrm{~F}$ & $\mathrm{C}_{6} \mathrm{H}_{5}$ & 91 \\
\hline 5 & $5 e$ & $p-\mathrm{CH}_{3}-\mathrm{C}_{6} \mathrm{H}_{4}$ & $\mathrm{C}_{6} \mathrm{H}_{5}$ & 90 \\
\hline 6 & $5 f$ & $p-\mathrm{OCH}_{3}-\mathrm{C}_{6} \mathrm{H}_{4}$ & $\mathrm{C}_{6} \mathrm{H}_{5}$ & 90 \\
\hline 7 & $5 \mathrm{~g}$ & $p-\mathrm{OH}-\mathrm{C}_{6} \mathrm{H}_{4}$ & $\mathrm{C}_{6} \mathrm{H}_{5}$ & 92 \\
\hline 8 & $5 \mathrm{~h}$ & $p-\mathrm{NO}_{2} \mathrm{C}_{6} \mathrm{H}_{4}$ & $\mathrm{C}_{6} \mathrm{H}_{5}$ & 81 \\
\hline 9 & $5 i$ & 2,4-di Cl- $\mathrm{C}_{6} \mathrm{H}_{3}$ & $\mathrm{C}_{6} \mathrm{H}_{5}$ & 83 \\
\hline 10 & $5 \mathbf{j}$ & 2,4,5-tri $\mathrm{OMeC}_{6} \mathrm{H}_{2}$ & $\mathrm{C}_{6} \mathrm{H}_{5}$ & 84 \\
\hline 11 & $5 \mathbf{k}$ & $2,4,5$-tri $\mathrm{OMeC}_{6} \mathrm{H}_{2}$ & $p-(\mathrm{OMe})-\mathrm{C}_{6} \mathrm{H}_{4}$ & 85 \\
\hline 12 & 51 & $p-\mathrm{CF}_{3}-\mathrm{C}_{6} \mathrm{H}_{4}$ & $p-(\mathrm{OMe})-\mathrm{C}_{6} \mathrm{H}_{4}$ & 79 \\
\hline 13 & $5 \mathrm{~m}$ & 2,4-di $\mathrm{CF}_{3}-\mathrm{C}_{6} \mathrm{H}_{3}$ & $p-(\mathrm{OMe})-\mathrm{C}_{6} \mathrm{H}_{4}$ & 78 \\
\hline 14 & $5 n$ & 2,4-di $\mathrm{CF}_{3}-\mathrm{C}_{6} \mathrm{H}_{3}$ & $3,4-\mathrm{di}(\mathrm{OMe})-\mathrm{C}_{6} \mathrm{H}_{4}$ & 83 \\
\hline 15 & 50 & 2-Furyl & $\mathrm{C}_{6} \mathrm{H}_{5}$ & 88 \\
\hline 16 & $5 p$ & 2-Thiophenyl & $\mathrm{C}_{6} \mathrm{H}_{5}$ & 87 \\
\hline 17 & $5 q$ & 3-Pyridyl & $\mathrm{C}_{6} \mathrm{H}_{5}$ & 91 \\
\hline
\end{tabular}

${ }^{\mathrm{a}}$ Yields for isolated pure products

As shown in Table-2, this protocol can be excellently applied on aromatic aldehydes with both electron-withdrawing groups and electron-donating groups and it is well amenable for aromatic aldehydes only (Table-2, entries 1-17). The reactions were completed within 15-25 min and the pure products were isolated in high yields. The synthetic route is facile, convergent and allows easy placement of a variety of substituents on aromatic ring $\mathrm{A}$ and $\mathrm{B}$ (Fig. 3). In the reaction protocol equimolar amounts of 6-methyl, 4-hydroxy pyranone, aryl amines, aromatic aldehydes and pyridinium ylide were reacted in the presence of 0.1 equivalents of $\mathrm{NEt}_{3}$ in ethanol solvent under reflux conditions afforded $\mathbf{5 a}$ in good yield. The expected product was formed in good yield in absence of any other stereomeric impurities.

A probable mechanism for the formation of product is depicted in Scheme-II, which is similar to that of mechanism

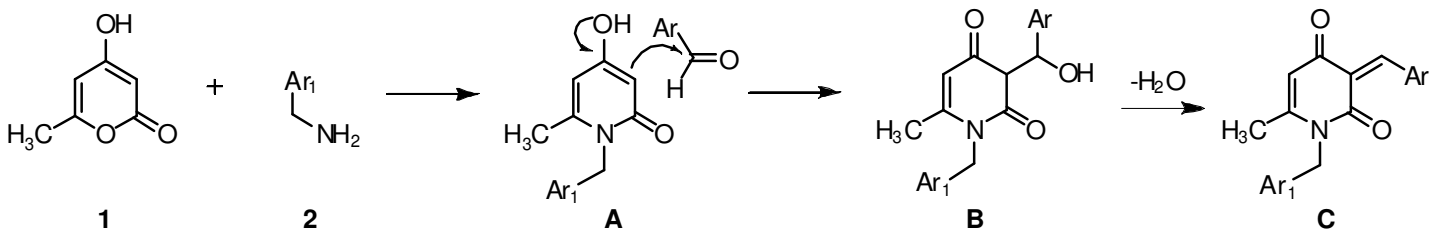<smiles>[R]C(=O)C[n+]1ccccc1</smiles><smiles>CCOC(=O)[C@@H]1Oc2cc(C)n(C[Al])c(=O)c2[C@H]1[Al]</smiles>

Scheme-II: Plausible mechanism for synthesis of tetrahydrofuro[3,2-c]pyridinone-2-carboxylate derivatives 
<smiles>CCOC(=O)[C@H]1Oc2cc(C)n(Cc3ccccc3)c(=O)c2[C@H]1c1ccccc1</smiles>

$5 a$<smiles>CCOC(=O)C1Oc2cc(C)n(Cc3ccccc3)c(=O)c2C1c1ccc(OC)cc1</smiles>

$5 f$<smiles>CCOC(=O)[C@H]1Oc2cc(C)n(Cc3ccc(OC)cc3)c(=O)c2C1c1cc(OC)c(OC)cc1OC</smiles>

$5 \mathrm{k}$<smiles>CCOC(=O)[C@H]1Oc2cc(C)n(Cc3ccccc3)c(=O)c2C1c1cccs1</smiles>

$5 p$

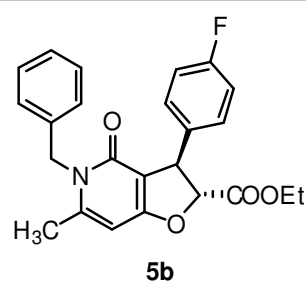<smiles>CCOC(=O)C1Oc2cc(C)n(Cc3ccccc3)c(=O)c2C1c1ccc(O)cc1</smiles>

$5 \mathrm{~g}$<smiles>CCOC(OCC)[C@H]1Oc2cc(C)n(Cc3ccc(OC)cc3)c(=O)c2C1c1ccc(C(F)(F)F)cc1</smiles>

51<smiles>CCOC(=O)[C@H]1Oc2cc(C)n(Cc3ccccc3)c(=O)c2C1c1cccnc1</smiles>

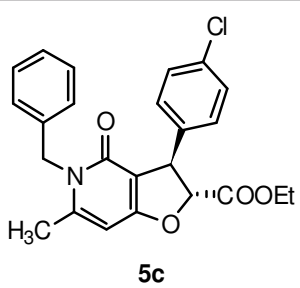<smiles>CCOC(=O)[C@H]1Oc2cc(C)n(Cc3ccccc3)c(=O)c2[C@H]1c1ccc([N+](=O)[O-])cc1</smiles>

$5 \mathrm{~h}$<smiles>CCOC(=O)[C@H]1Oc2cc(C)n(Cc3ccccc3)c(=O)c2[C@H]1c1ccc(Br)cc1</smiles><smiles>CCOC(=O)[C@H]1Oc2cc(C)n(Cc3ccccc3)c(=O)c2[C@H]1c1ccc(Cl)cc1Cl</smiles>

$5 i$<smiles>CCOC(=O)[C@H]1Oc2cc(C)n(Cc3ccc(OC)cc3)c(=O)c2C1c1ccc(C(F)(F)F)cc1C(F)(F)F</smiles>

$5 \mathrm{~m}$<smiles>CCOC(=O)[C@H]1Oc2cc(C)oc(=O)c2C1c1ccccc1</smiles>

$6 a$

$5 n$<smiles>CCOC(=O)[C@H]1Oc2cc(C)[nH]c(=O)c2C1c1ccccc1</smiles>

$6 b$

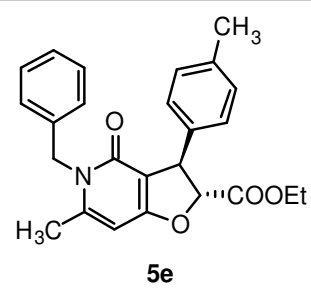<smiles>CCOC(=O)[C@H]1Oc2cc(C)n(Cc3ccccc3)c(=O)c2C1c1cc(OC)c(OC)cc1OC</smiles>

$5 j$<smiles>CCO[C@H]1Oc2cc(C)n(Cc3ccc(OC)c(OC)c3)c(=O)c2C1c1ccc(C(F)(F)F)cc1C(F)(F)F</smiles><smiles>CCO[C@H]1Oc2cc(C)n(Cc3ccccc3)c(=O)c2C1c1ccco1</smiles>

50

Fig. 3. Synthesis of SAR directed a combinatorial library of tetrahydrofuro[3,2-c]pyridinone-2-carboxylate derivatives

of dihydrofuropyrazole synthesis. Further the reaction occurs via an initial Knoevenagel condensation between 6-methy, 4hydroxy pyranone $\mathbf{A}$ and aromatic aldehyde $\mathbf{3}$ to give the intermediate $\mathbf{C}$. On the other hand, the pyridinium ylide $\mathbf{4}$, which forms from the reaction of $\mathrm{N}$-phenacyl pyridinium bromide $\mathbf{3}$ with triethyl amine undergoes Michael addition with an intermediate $\mathbf{D}$ to afford the enolate intermediate $\mathbf{E}$. The enolate $\mathbf{E}$ eliminates pyridine and cyclizes instantly to give dihydrofuro[1,2-b]pyrazole $\mathbf{5}$.

Anti-inflammatory activity: All the synthesized compounds were evaluated for anti-inflammatory (TNF- $\alpha$, IL-6, COX-1 and COX-2, $\beta$-glucuronidase and trypsin activity). TNF- $\alpha$, IL$6, \mathrm{COX}-1$ and $-2, \beta$-glucuronidase and trypsin inhibition assays are prepared according to the literature procedure [27-29]. Anti-inflammatory activity against TNF- $\alpha$ and IL- 6 and results are summarized in Table- 3 . Results revealed that almost all the synthesized compounds $\mathbf{5 a}$ to $\mathbf{5 q}$ have shown significant activity against of TNF- $\alpha$ (68-38\%) as compared with standard dexamethasone (72\%). Overall, compounds 5j, 5k, 5l, 5m and $\mathbf{5 n}$ are the most active among the series against TNF- $\alpha$ and have least cytotoxicity against CCK- 8 cell line. The structure activity relationship study in these compounds revealed that compounds containing electron-withdrawing substituent on aromatic ring A have higher TNF- $\alpha$ inhibition than their counterpart containing electron-donating substituent on aromatic ring B. Bioisosteric replacement of aromatic ring by furan, thiophene, pyridine there is slight decrease in TNF- $\alpha$ inhibition. When we

\begin{tabular}{cccc}
\multirow{2}{*}{$\begin{array}{c}\text { TABLE-3 } \\
\text { ANTI-INFLAMMATORY ACTIVITY AGAINST TNF- } \alpha \text { AND IL-6 }\end{array}$} \\
\hline \multirow{2}{*}{ Compounds } & \multicolumn{3}{c|}{ Inhibition (\%) at $10 \mu \mathrm{M}$} \\
\cline { 2 - 4 } & TNF- $\alpha$ & IL-6 & Toxicity \\
\hline $\mathbf{5 a}$ & 44 & 52 & 53 \\
$\mathbf{5 b}$ & 48 & 50 & 52 \\
$\mathbf{5 c}$ & 42 & 48 & 56 \\
$\mathbf{5 d}$ & 38 & 56 & 46 \\
$\mathbf{5 e}$ & 39 & 57 & 48 \\
$\mathbf{5 f}$ & 41 & 58 & 43 \\
$\mathbf{5 g}$ & 37 & 61 & 44 \\
$\mathbf{5 h}$ & 38 & 41 & 53 \\
$\mathbf{5 i}$ & 31 & 43 & 56 \\
$\mathbf{5 j}$ & 51 & 78 & 25 \\
$\mathbf{5}$ & 56 & 81 & 22 \\
$\mathbf{5 l}$ & 68 & 83 & 24 \\
$\mathbf{5 m}$ & 54 & 76 & 30 \\
$\mathbf{5 n}$ & 58 & 70 & 38 \\
$\mathbf{5 0}$ & 39 & 47 & 47 \\
$\mathbf{5 p}$ & 38 & 48 & 41 \\
$\mathbf{5 q}$ & 41 & 47 & 43 \\
$\mathbf{5 q}$ & 72 & 94 & 00 \\
\hline
\end{tabular}

The results summarized are the mean values of $n=2$

compared these results with its siblings, the newly synthesized pyridone derivatives shows promising activity against of all TNF- $\alpha$ and IL-6, COX-2, $\beta$-glucuronidase enzymes than $6 \mathbf{a}$ and $\mathbf{6 b}$ compounds. 
The synthesized compounds screened for IL-6 inhibition and results are summarized in Table- 3 . Results showed that synthesized compounds are found to be active against IL-6. Compounds $\mathbf{5 a}$ to $\mathbf{5 q}$ have shown significant inhibition against of IL-6 (98-95 \%) as compared with standard dexamethasone (94\%). Overall, compounds $\mathbf{5 j}$, 5k, 5l, 5m, 5n are most active and have lowest cytotoxicity against CCK-8 cell line. Compounds with fluorine and trifluoromethyl substitution have highest activity than the compounds containing chloro and bromo substitution on aromatic ring $\mathbf{A}$.

The synthesized compounds are subjected to COX inhibition study and results are shown in Table-4. Compounds $\mathbf{5 j}$, 5l, 5m, 5n and 5n have shown potent (89.25-83.28 \%) COX-2 inhibition. COX-2 is expressed during inflammation and COX1 is constitutive, which is involved in physiological functions. Therefore, compounds selectively inhibit the COX-2 have good account of anti-inflammatory activity. Compounds under investigation have inhibited COX-2 preferentially and are more selective towards COX-2 rather than COX-1. Structure-activity relationship study with respect to cyclooxygenase inhibition revealed that compounds containing $\mathrm{OMe}$ and $\mathrm{CF}_{3}$ groups shows better activity.

\begin{tabular}{ccccc}
\multicolumn{5}{c}{ TABLE-4 } \\
\multicolumn{5}{c}{$\begin{array}{c}\text { ANTI-INFLAMMATORY ACTIVITY OF NOVEL } \\
\text { DERIVATIVES AT 1 } \mu \text { CONCENTRATION }\end{array}$} \\
\hline Compd. & COX-1 & COX-2 & Trypsin & $\beta$-Glucuronidase \\
& $(\%)$ & $(\%)$ & $(\%)$ & $(\%)$ \\
\hline $\mathbf{5 a}$ & 38.41 & 60.37 & 50.85 & 44.32 \\
$\mathbf{5 b}$ & 43.43 & 76.54 & 56.33 & 63.46 \\
$\mathbf{5 c}$ & 37.14 & 59.46 & 48.13 & 43.87 \\
$\mathbf{5 d}$ & 36.14 & 57.11 & 48.38 & 40.87 \\
$\mathbf{5 e}$ & 37.22 & 58.34 & 47.45 & 41.25 \\
$\mathbf{5 f}$ & 40.13 & 62.47 & 52.76 & 43.53 \\
$\mathbf{5 g}$ & 39.18 & 60.55 & 51.23 & 42.78 \\
$\mathbf{5 h}$ & 42.69 & 74.32 & 59.83 & 62.15 \\
$\mathbf{5 i}$ & 40.25 & 54.78 & 46.72 & 40.43 \\
$\mathbf{5 j}$ & 44.25 & 79.84 & 64.43 & 71.65 \\
$\mathbf{5 k}$ & 46.86 & 82.37 & 68.56 & 73.58 \\
$\mathbf{5 1}$ & 53.32 & 83.89 & 69.08 & 74.24 \\
$\mathbf{5 m}$ & 57.68 & 85.15 & 69.86 & 75.03 \\
$\mathbf{5 n}$ & 59.63 & 86.78 & 71.34 & 75.82 \\
$\mathbf{5 0}$ & 38.12 & 50.76 & 49.47 & 42.93 \\
$\mathbf{5 p}$ & 37.78 & 51.39 & 48.64 & 40.15 \\
$\mathbf{5 q}$ & 39.13 & 49.38 & 50.18 & 43.19 \\
$\mathbf{5 r}$ & 10.43 & 11.64 & 09.11 & 06.05 \\
$\mathbf{5 s}$ & 12.75 & 13.54 & 11.90 & 09.47 \\
\hline ASA & - & 36.93 & - & - \\
SC 560 & 36.71 & - & - & - \\
SA & - & - & 84.99 & 24.77 \\
\hline The results are summarized are the mean values of $\mathrm{n}=2$ \\
ASA = Acetyl salicylic acid, SC 560 a standard COX-1 \\
\hline
\end{tabular}

Trypsin is a member of the serine proteases family. These proteases are involved in initiation of inflammation; moreover, serine protease inhibition has been considered as one of the target for design of anti-inflammatory drugs [30]. From Table4, compounds 5j (64.43\%), 5k (68.56 \%), 5l (69.08\%), 5m $(69.86 \%), \mathbf{5 n}(71.84 \%)$ have shown good trypsin inhibition as compared with standard salicylic acid ( $84.99 \%)$. The enzyme $\beta$-glucuronidase has been considered as one of the targets in the design of anti-inflammatory agents as it play the role in the initiation of inflammation. The lysosomes of the polymorphonuclear neutrophils are rich in $\beta$-glucuronidase. This enzyme is attributed as one of the mediators for initiating the process of inflammation. Compounds $\mathbf{5 j}$, 5k, 5l, 5m and $\mathbf{5 n}$ have shown excellent inhibition of $\beta$-glucuronidase (75.82-71.65\%) as compared with standard salicylic acid (24.77\%).

\section{Conclusion}

In conclusion we have developed a facile, ecofriendly methodology for synthesis of tetrahydrofuro[3,2-c]pyridine2-carboxylate derivatives by one pot multi-component condensation of 6-methyl, 4-hydroxy pyranone, aryl amines, aromatic aldehydes and pyridenium ylied, in presence of triethylamine as a catalyst under ethanol reflux conditions. Anti-inflammatory activity study of newly synthesized compounds shows that compounds $\mathbf{5 j}, \mathbf{5 k}, \mathbf{5 l}, \mathbf{5 m}$ and $\mathbf{5 n}$ were excellent inhibitors of TNF- $\alpha$, IL-6, COX-2, trypsin and $\beta$-glucuronidase.

\section{ACKNOWLEDGEMENTS}

One of the authors, (VST) thanks to CSIR-Indian Institute of Chemical Technology and University of Hyderabad, Hyderabad, India for recording NMR, Mass, HRMS spectra and biological evolution of samples.

\section{REFERENCES}

1. I. Bradiakova, T. Durcekova, N. Pronayova, A. Gatial and A. Krutosikova, Chem. Pap., 63, 586 (2009); https://doi.org/10.2478/s11696-009-0052-4.

2. Y. Sugie, S.J. Truesdell, J.W. Wong, N. Yoshikawa and A. Sugiura, Furopyridine Antibacterials and Production Thereof, EP 999212 A1 (2000).

3. T. Fukuda, Y. Yamaguchi, R. Masuma, H. Tomoda and S.J. Omura, Antibiot., 58, 309 (2005);

https://doi.org/10.1038/ja.2005.38.

4. B. Wolters and U. Eilert, Planta Med., 43, 166 (1981); https://doi.org/10.1055/s-2007-971494.

5. G. Petit-Pali, M. Rideau and J.C. Chenieux, Planta Med. Phytother., 16, 55 (1982).

6. G.H. Svoboda, G.A. Poore, P.J. Simpson and G.B. Boder, J. Pharm. Sci., 55, 758 (1966); https://doi.org/10.1002/jps.2600550803.

7. L.K. Basco, S. Mitaku, A.L. Skaltsounis, N. Ravelomanantsoa, F. Tillequin, M. Koch and J. Le Bras, Antimicrob. Agents Chemother., 38, 1169 (1994); https://doi.org/10.1128/AAC.38.5.1169.

8. D.L.J. Clive and X. Huang, J. Org. Chem., 69, 1872 (2004); https://doi.org/10.1021/jo030284g.

9. Y. Rok Lee, B.S. Kim and H. Il Kweon, Tetrahedron, 56, 3867 (2000); https://doi.org/10.1016/S0040-4020(00)00307-0.

10. H. Senboku, M. Takashima, M. Suzuki, K. Kobayashi and H. Suginome, Tetrahedron, 52, 6125 (1996); https://doi.org/10.1016/0040-4020(96)00210-4.

11. H. Suginome, K. Kobayashi, M. Itoh, S. Seko and A. Furusaki, J. Org. Chem., 55, 4933 (1990); https://doi.org/10.1021/jo00303a034.

12. B.B. Snider and Q. Che, Org. Lett., 6, 2877 (2004); https://doi.org/10.1021/o1049130t.

13. D. Conreaux, T. Delaunay, P. Desbordes, N. Monteiro and G. Balme, Tetrahedron Lett., 50, 3299 (2009); https://doi.org/10.1016/j.tetlet.2009.02.073.

14. M.C. Pirrung and F. Blume, J. Org. Chem., 64, 3642 (1999); https://doi.org/10.1021/jo982503h.

15. J. Su, J. Xiong, S. Liang, G. Qiu, X. Feng, H. Teng, L. Wu and X. Hu, Synth. Commun., 36, 693 (2006); https://doi.org/10.1080/00397910500446530.

16. R. Zhang, Y. Liang, G. Zhou, K. Wang and D. Dong, J. Org. Chem., 73, 8089 (2008); https://doi.org/10.1021/jo801289p. 
17. F. Liang, S. Lin and Y. Wei, J. Am. Chem. Soc., 133, 1781 (2011); https://doi.org/10.1021/ja110870f.

18. S. Tangeti, G.V. Siva Prasad, J. Panda and K.R. Varma, Synth. Commun., 46, 878 (2016); https://doi.org/10.1080/00397911.2016.1174781.

19. H.S.P. Rao and V.S. Tangeti, J. Chem. Sci., 125, 777 (2013); https://doi.org/10.1007/s12039-013-0458-y.

20. H.S.P. Rao, V.S. Tangeti and L.N. Adigopula, Res. Chem. Intermed., 42, 7285 (2016); https://doi.org/10.1007/s11164-016-2536-5.

21. V.S. Tangeti, R. Varma K, G.V. Siva Prasad and K.V.V.V. Satyanarayana, Synth. Commun., 46, 613 (2016);

https://doi.org/10.1080/00397911.2016.1159696.

22. H. S.P. Rao and V. S. Tangeti, Lett. Org. Chem., 9, 218 (2012); https://doi.org/10.2174/157017812800167501.

23. H.S.P. Rao and V.S. Tangeti, Proc. Natl. Acad. Sci. India Sect. A: Phys. Sci., 85, 41 (2015); https://doi.org/10.1007/s40010-014-0179-8.
24. N.S. Topno, H.S.P. Rao, V.S. Tangeti and R. Krishna, Acta Crystallogr., E69, o284 (2013); https://doi.org/10.1107/S1600536812051872.

25. V.S. Tangeti, D. Vasundhara, K.V.V.V. Satyanarayana and K.S.P. Kumar, Asian J. Chem., 29, 1525 (2017); https://doi.org/10.14233/ajchem.2017.20550.

26. S.M. Rajesh, S. Perumal, J.C. Menéndez, S. Pandian and R. Murugesan, Tetrahedron, 68, 5631 (2012); https://doi.org/10.1016/j.tet.2012.04.058.

27. C. Hwang, M. Catanaga, A. Gale and T. Gatanaga, J. Immunol., 151, 5631 (1993).

28. M. Tandon, P. Tandon, J.P. Barthwal, T.N. Bhalla and K.P. Bhargava, Drug Res., 32, 1233 (1982).

29. B.P. Bandgar, B.S. Hote, S.S. Jalde and R.N. Gacche, Med. Chem. Res., 21, 3006 (2012); https://doi.org/10.1007/s00044-011-9834-7.

30. T. Bilfinger and G. Stefano, Curr. Pharm. Des., 8, 505 (2002); https://doi.org/10.2174/1381612023395763. 